\title{
Lying Posture Classification for Pressure Ulcer Prevention
}

\author{
Aung Aung Phyo Wai ${ }^{1}$, Siang Fook Foo ${ }^{1}$, Weimin Huang ${ }^{1}$, Jit Biswas ${ }^{1}$, \\ Chi-Chun Hsia ${ }^{2}$, Koujuch Liou ${ }^{2}$ and Philip Yap ${ }^{3}$ \\ ${ }^{1}$ Institute for Infocomm Research, 1 Fusionopolis Way \#21-01 Connexis, \\ Singapre 138632.\{apwaung, sffoo,wmhuang, biswas\}@i2r.a-star.edu.sg \\ ${ }^{2}$ Industrial Technology Research Institute, ITRI South, No. 31, Gongye $2^{\text {nd }}$ Rd., \\ Annan District, Tainan City 709, Taiwan R.O.C \{shiacj, lioukj\}@itri.org.tw \\ ${ }^{3}$ Alexandra Hospital, 378, Alexandra Road, Singapore 159964. \\ Philip_YAP@Alexhosp.com.sg
}

\begin{abstract}
Pressure ulcers are a common problem among patients with limited mobility, such as those bedbound and wheelchair-bound. Constant and prolonged applied pressure is one of the extrinsic factors contributing to the development of pressure ulcers. Analyzing lying postures together with interface pressure measurements from a pressure sensitive bed helps revealing pressure hot spots that can potentially lead to pressure ulcer development. We propose an intelligent system that features lying posture classification with pressure hot spots identification based on interface pressure measurements to possibly identify potential pressure ulcer risk and to provide effective preventive measures. Experimental outcomes correctly classify different lying postures with an accuracy of up to $93 \%$. The proposed system is expected to assist caregivers to detect risk evidence and to provide timely and appropriate interventions for effective pressure ulcer prevention.
\end{abstract}

Keywords: pressure sensitive bed; pressure hot spots; lying posture classification; interface pressure; pressure ulcer prevention

\section{INTRODUCTION}

Rapid growth of elderly population is one of the most pressing issues in healthcare today. For elderly, especially those who are frail with compromised independence, their safety and quality of care can be enhanced by providing continuous surveillance to monitor their health status and well being. This form of improved care can be facilitated with Information-Communication-Technologies-(ICT)-enabled personal health monitoring solutions which leverage on ambient-assisted living environment using sensors, actuators and robotics. This new healthcare paradigm is widely accepted as a viable solution in various healthcare settings $[1,2]$. 
The elderly who are frail and disabled spend most of their time in bed and usually require around-the-clock care from caregivers. As a result of prolonged recumbency, those who are immobile face the risk of developing pressure ulcers [3, 4]. Pressure ulcer, which is an area of localized damage to the skin and to the underlying tissues caused by pressure, shear and/or friction, is a common and important issue to be addressed [5]. The current understanding of pressure ulcers confines the definition only to deep tissue injuries caused by sustained deformations of subcutaneous soft tissues in the vicinity of bony prominences [6]. About $11 \%$ of nursing home residents in the U.S suffer from pressure ulcer related problems [7]. Also, epidemiological studies in the UK reported pressure ulcer prevalence ranging from $4.4 \%$, in a community unit, up to $37 \%$ in palliative care [8]. The cost of managing pressure ulcers is enormous and estimated by the UK National Health System to be in the range of 1.4 to 2.1 billion pounds annually, with most of the cost attributed to nursing time [9]. According to a recent survey [10], pressure ulcer is still a major and costly healthcare issue in care institutions due to the inability to provide timely preventive measures. Hence, the importance of early recognition of potential risk factors and timely interventions to alleviate this problem must be emphasized.

With advances in pressure sensing technologies, pressure sensing devices can measure interface pressure [11] exerted on a subject unobtrusively and continuously $[12,13]$. Interface pressure measurements are usually analyzed offline [14, 15] to evaluate and assess the pressure relieving capabilities of different support surfaces and devices. On the other hand, real-time interface pressure measurements are utilized to provide the interventions of turning the subjects and positioning appropriate support surfaces $[16,17]$. However, measured interface pressure and pressure ulcer do not have a very high qualitative relationship [18, 19]. Although interface pressure alone has inherent limitations to identify pressure ulcers development, proper interpretation and processing techniques of analyzing interface pressure measurements may assist in effective prevention of pressure ulcers $[16,17]$. Our approach for pressure ulcer prevention is conceptually based on continuous interface pressure measurements identifying potential pressure hot spots on vulnerable areas of the body. This allows caregivers to have a better understanding of pressure ulcer risks, thus enabling to implement timely and appropriate preventive measures. For example, mapping appropriate interface pressure parameters with recognized lying position/posture as shown in Figure 1, the system can provide the caregivers an understanding and assessment of possible pressure ulcer risks of the subject. In the event of a high risk of pressure ulcer development, the caregivers can be notified through in-situ alerts and reminders. Although we have targeted our solution to pressure ulcer prevention, the recognized features and contexts of pressure evidences can be applicable to other bedbased health and well-being monitoring applications.

The objective of this work is to present and to test an intelligent system that features lying posture classification with pressure hot spots identification based on interface pressure measurements to possibly identify potential pressure ulcer risks. The paper is organized as follows: in section 2, we provide literature survey on various pressure ulcer prevention approaches and the rationale for pressure ulcer prevention using the 

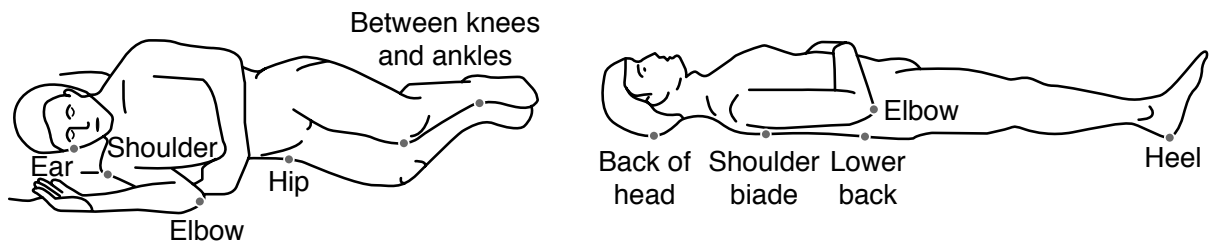

Figure 1. Common pressure-ulcer-prone locations associated with different lying postures.

pressure sensitive bed. The sensing hardware and system architecture of our pressure ulcer prevention pilot testbed, intelligent BEdsore Prevention System (iBEPS), are discussed in section 3. Section 4 provides details of the proposed lying posture classification approach and various methodologies. Detailed analyses of different lying posture classification approaches with experimental results, limitations and future directions are presented in sections 5 and 6 , respectively.

\section{RATIONALE FOR PRESSURE ULCER PREVENTION}

The risk factors for pressure ulcer development can be broadly classified into intrinsic and extrinsic factors. Intrinsic factors are comprised of parameters specific to the human subject such as body weight, immobility, sensory perception and nutrition. Extrinsic factors refer to environmental conditions such as intensity and duration of interface pressure, friction, shear force and degree of skin exposure to moisture. Intrinsic factors are not usually easily remediable in the short term, whereas extrinsic factors are more modifiable and of these, the magnitude and duration of interface pressure is deemed to be of utmost importance [20]. Both Reswick and Rogers pressure-time curve [21] and Sigmoid injury threshold [22] for pressure ulcer risks illustrate this point properly. Therefore, interventions to decrease the risks of pressure ulcers should aim at reducing the magnitude and duration of the applied pressure exerted on the subject's body parts. To fulfill this requirement, the potential risk evidence caused by the applied pressure on the pressure hot spots must be detected and identified continuously through a pressure sensing device [12].

\subsection{Pressure Ulcer Assessment and Prevention Approaches}

Although there are several guidelines, assessment skills, and approaches for pressure ulcers prevention [23, 24], it is still an unresolved issue in practical nursing care environments. Risk assessment skills on specific subjects were studied to validate the applicability of existing risk measures [24, 25] but performing accurate risk assessment to individual subject is still a challenging task. Defloor et al. evaluated different operating-table mattresses to determine the most suitable support surface for pressure ulcer prevention [26]. Their study revealed that there were apparent differences in interface pressure for different positions of the subject, and no mattress could reduce applied pressure sufficiently. A review on different support surfaces shows that pressure ulcer development also depends on the types of materials used for supporting surfaces, but 
none of them is able to fully prevent pressure ulcers [27]. Turning of the patient has been shown to be a feasible method of reducing pressure ulcers, but the effect of posture change during the turning interval has not been considered [28]. Evaluation of interface pressure on pressure ulcers prevention with pressure sensors [12] proved that body turning or repositioning, either voluntarily [17] or based on continuous pressure measurements, could reduce the risks of developing pressure ulcers.

Air-filled mattresses [29] are commonly used for relieving applied pressure on the body and have been shown effective [30]. However, the actuation (filling/releasing air) is typically performed either randomly or based on a simplified human model [29]. It does not determine the potential risk locations associated with a lying posture or the risk of developing pressure ulcers at those locations. From the biomechanical perspective, the prevention of ulcers caused by internal tissues load has been studied, and the effect of high internal load but low external load that causes tissue deformations has been demonstrated [31,32]. Actimeter and elastography were employed to observe activity levels and to monitor tissue deformation, but they could only observe limited areas of the body and could not measure the effect of applied pressure on human body [33]. A portable gauge measuring skin biomechanical properties was developed to detect tissue deformation or injury, but could not measure applied load exerted on different areas of the body [34]. Although the risks of pressure ulcers can potentially be reduced through various approaches, it is still difficult to provide effective preventive measures due to the complex interplay of multiple intrinsic and extrinsic factors associated to pressure ulcer development.

\subsection{Pressure Sensitive Bed for Pressure Ulcer Prevention}

The constant and prolonged applied pressure over bony prominences is one of the extrinsic contribution factors of developing pressure ulcers [35]. However, considering interface pressure measurements alone may not be able to prevent pressure ulcers completely [19]. Not all commonly used and well-known interface pressure parameters can provide reliable representation of pressure ulcer risk evidence [18]. Recent research has also pointed out that pressure ulcers can still occur due to high internal tissue load or stress even if the interface pressure is low $[31,32]$. However, as tissue tolerance is still an undetermined parameter, the best solution for now is to identify high interface pressure points, viz. pressure hot spots, and to facilitate desirable pressure changes at the earliest stage. By profiling interface pressure with body position/posture continuously over time, the potential pressure hot spots at critical locations of the patient's body can be determined. According to the identified pressure hot spots and pressure distribution with the lying posture, potential pressure ulcer risks may be recognized accurately. This helps caregivers to provide timely and appropriate interventions to relieve the applied pressure from the identified pressure hot spots.

Decreasing the excessive applied pressure over bony prominences and avoiding prolonged body rest are currently well-known strategies for pressure ulcer prevention $[23,35]$. Ideally, if caregivers know where the potential risk locations are, they can implement the interventions such as relieving pressure, changing lying posture, providing support surfaces, applying medications, etc. [5, 28], thereby decreasing the 


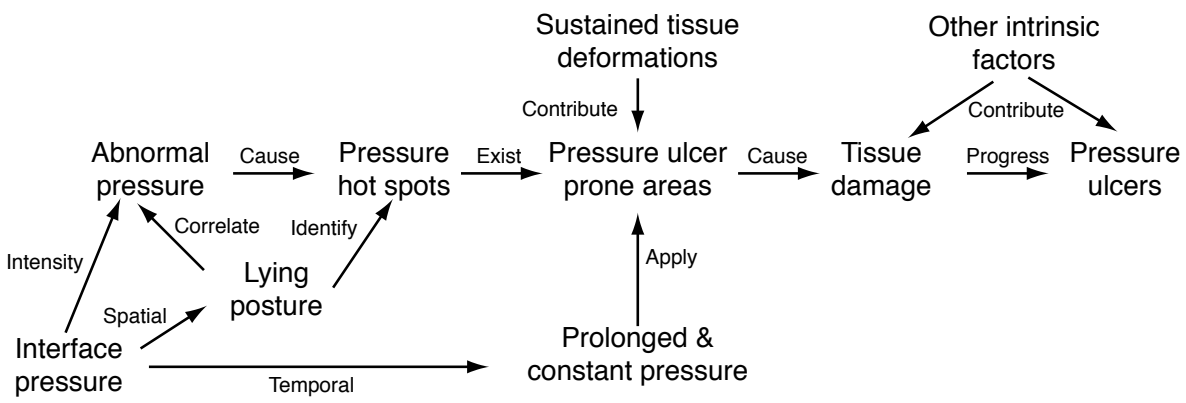

Figure 2. From interface pressure to pressure ulcer development.

likelihood of pressure ulcers development. Knowledge of high risk locations can be determined by employing a pressure sensitive bed to continuously monitor the potential risk evidences (pressure hot spots) over bony prominences [12, 13]. Reenalda et al. demonstrated that shifting in sitting posture might reduce the pressure ulcer development as this process increases tissue oxygenation [17]. Our work illustrates the possibility of an intelligent and personalized means of identifying potential pressure ulcer risks from interface pressure measurements with pressure hot spots identification using a pressure sensitive bed. Figure 2 delineates how interface pressure causes development of pressure ulcer. The mapping or association of interface pressure measurements with body position/posture provides information about the locations of pressure hot spots. In this paper, we present a two-level lying posture classification approach with experimental analysis of different posture classification methods. In our ongoing work, we are examining the aspect of mapping identified hot spots to the posture through the use of three-dimensional computer graphics modeling techniques.

\section{PILOT TESTBED FOR INTELLIGENT BEDSORE PREVENTION SYSTEM: iBEPS}

The purpose of iBEPS is to provide a system for continuous monitoring of applied pressure, thereby enabling continuous assessment of potential pressure ulcer risks. It is important from clinical perspective that the system is cost-effective and it should be easily integrated with other preventive measures [23].

\subsection{Hardware Configuration}

An array of piezo-resistive pressure sensors called Force Sensing Resistors (FSR, Interlink Electronics, Camarillo, CA, USA) was configured in a manner similar to the Tekscan Flexiforce sensor (Tekscan Inc, Boston MS, USA) to determine both spatial and temporal pressure evidences of the subject. According to the sensor's technical specifications and our evaluation experiments, the sensor exhibits non-linearity of $3 \%$, repeatability of $\pm 3 \%$ of full scale, accuracy with variation of $\pm 5 \%$, and has a response time of 1 to $2 \mathrm{msec}$. The acquired pressure data are fed into relevant processing 
(a)

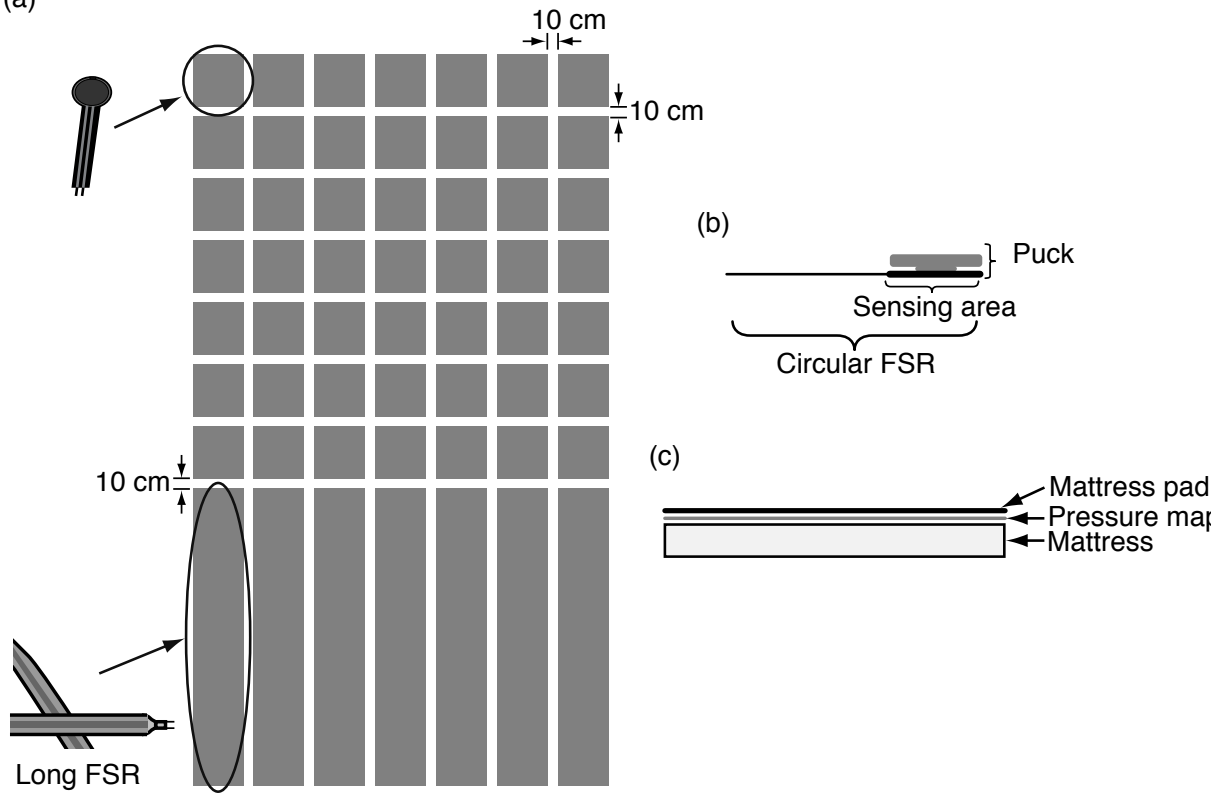

Figure 3. Custom-designed pressure sensitive bed configuration. (a) Pressure sensor layout. (b) Pressure sensor packaging. (c) Pressure map usage.

modules to extract features, to classify postures, and to detect movements of the patient lying on the bed. Figure 3 illustrates how pressure sensors are packaged and configured on a bed of $1.8 \mathrm{~m}^{2}$ area. The spacing between adjacent sensors, both circular and long FSRs, is about $10 \mathrm{~cm}$. In order to achieve high sensitivity in measuring the applied normal pressure, a puck is attached to the sensing area of each circular FSR as shown in Figure 3(b). The pressure map, a sheet that contains matrix of pressure sensors, is sandwiched between the thin mattress pad and underlying thick mattress as shown in Figure 3(c).

The sensing bed layout consists of a total of 56 sensors including 49 circular FSRs and 7 long FSRs as shown in Figure 3(a). The analog readings from these sensors are sampled from $1.0 \mathrm{~Hz}$ to $10.0 \mathrm{~Hz}$. The readings are converted into digital representation ranging from 0 to 1023 (10 bits resolution) through data acquisition units. The applied load to each FSR is then converted into weight in kilograms, $\mathrm{W}_{\mathrm{kg}}$, from acquired data, $\mathrm{x}$, according to FSR applied load-voltage characteristics provided by the manufacturer after conditioning and calibrating the sensors using Equation (1):

$$
W_{k g}(x)=\frac{\left.10^{\left(\frac{\log _{10}\left(\frac{10 x}{1024-x}\right)}{-0.7838}+3.186\right.}\right)}{1000.00}
$$


Subsequently, the weight is used to calculate interface pressure $\left(\mathrm{kg} / \mathrm{cm}^{2}\right)$ based on the coverage area of each sensor. The weight distribution of the pressure sensitive bed is used for lying posture classification.

\subsection{System Architecture and Development}

A basic functionality of iBEPS is continuous sensing of interface pressure exerted on the subject. Its extended functionalities can range from recognizing the risk evidences from interface pressure measurements to supporting prompts and reminders for timely intervention. As shown in Figure 4, the pressure sensors on the bed measure the applied pressure exerted on the subject. The controller is mainly responsible for acquiring data from sensors and converting them into meaningful interface pressure parameters. The analyzer performs processing of measured pressure data and provides automated personalized intelligence to recognize risks associated with pressure ulcers. It interacts with various reminder components that provide desirable audible and visual reminders to notify the caregivers when potential risks or undesirable events occur to the patients. The database server provides a unified storage for the whole system. The application server enables seamless access, and either local or remote management of the system. All components of iBEPS, except sensors and reminders, are software-based. A major challenge addressed in iBEPS is to reduce uncertainty due to noisy pressure sensor data, ensuring that the automated intelligence provides sufficiently reliable and robust outcomes.

\subsection{Pressure Ulcer Prevention Process}

Figure 5 illustrates the step-by-step operations and processes involved in pressure ulcer risk recognition using iBEPS. By correlating and mapping the subject's position or posture with interface pressure parameters, potential pressure hot spots can be identified

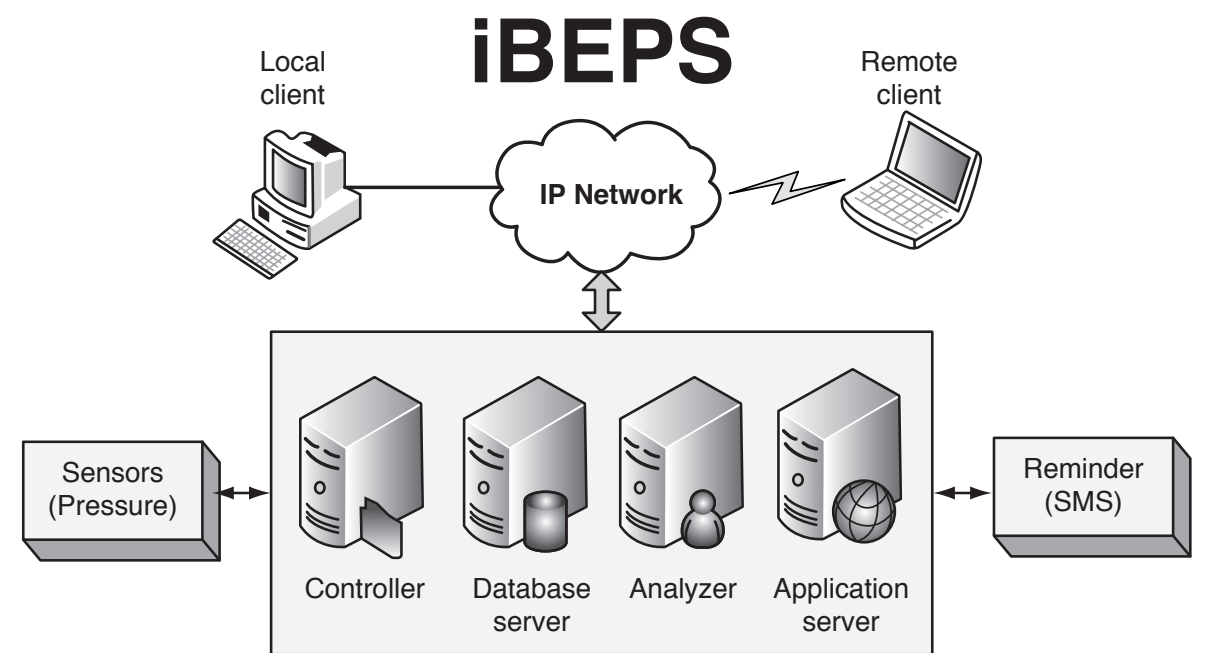

Figure 4. iBEPS system architecture and components. 


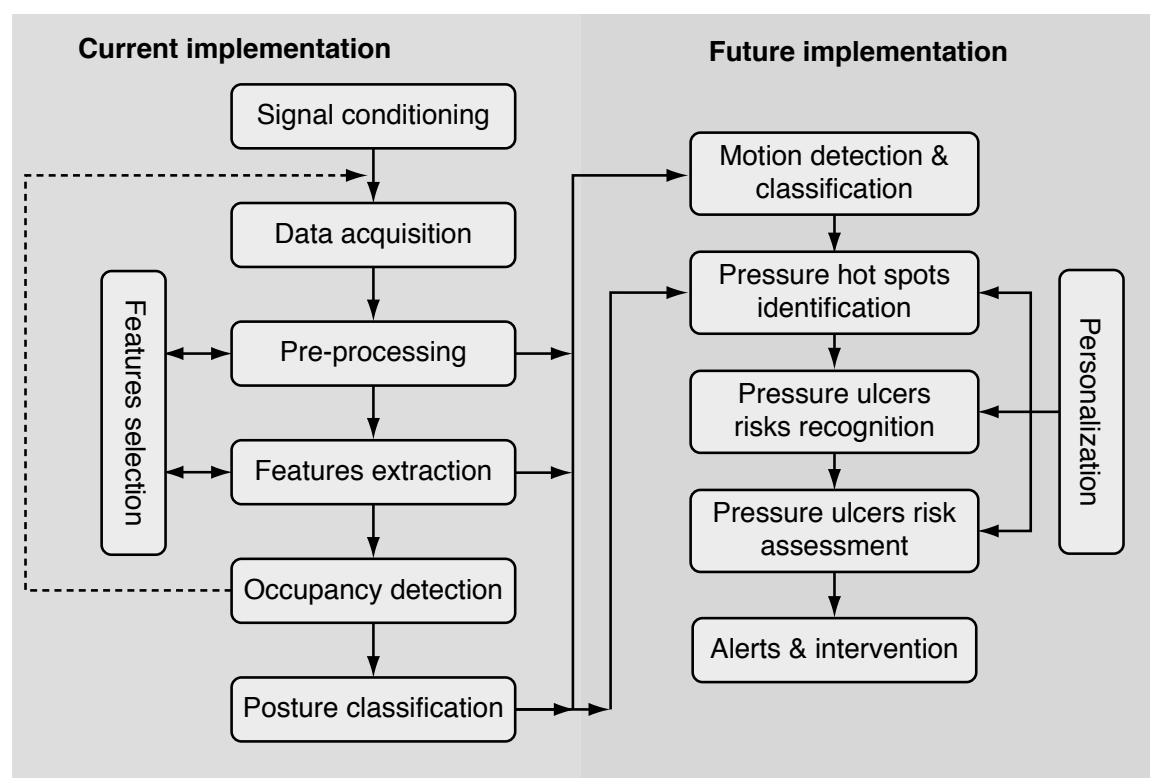

Figure 5. Step-by-step pressure ulcer prevention processes.

continuously. The intelligent analysis of pressure measurements can also extend to identify the subject's movements, to observe the repositioning or changes in pressure distribution, and to determine the associated pressure relieves caused by such movements. The intelligent algorithms carry out data/information fusion based on various identified pressure evidences [18], prior pressure ulcer risks, as well as personal profiles. The potential pressure ulcer risks of the patients are then estimated based on measured pressure evidences and patient's specific parameters. Finally, appropriate alerts and reminders are sent to the caregivers who should promptly respond to the patients according to recognized pressure ulcer risks. As depicted in Figure 5, pressure ulcer prevention process includes two parts: (a) an automated classification of the lying posture into one of the canonical lying postures, and (b) the use of interface pressure measurements to predict potentially at risk hot-spots in each lying posture. The current implementation includes accurate and reliable lying posture classification, whereas future implementation will involve from personalized pressure hot spots identification to pressure ulcer risks recognition and notification.

\section{APPROACH AND METHODOLOGIES FOR LYING POSTURE CLASSIFICATION}

In addition to the lying posture classification of the subject, the interface pressure measurements can also be used to determine the duration and intensities of applied pressure to the subject's body. Based on the intensities and duration of interface pressure associated with the detected lying posture, pressure hot spots that may potentially lead to 


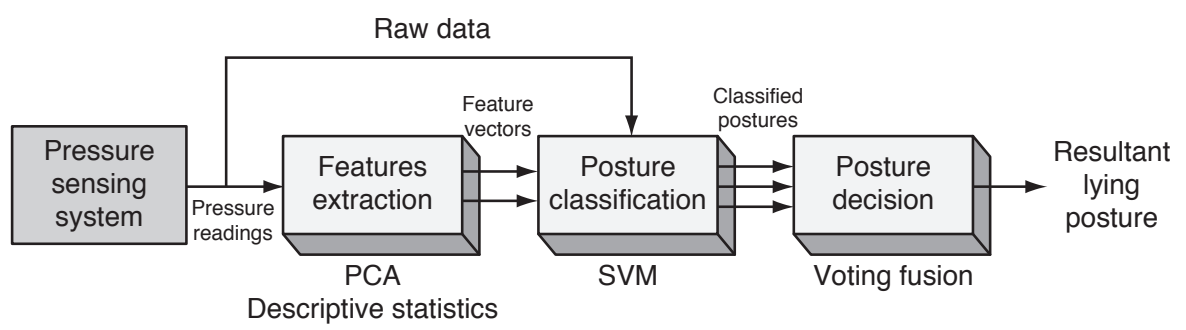

Figure 6. Two-level lying posture classification approach and methodologies.

development of pressure ulcers can be identified. Previous works on posture classification used different sensor configurations, and considered only coarse-grained lying postures $[36,37]$. In our work, a two-level classification approach is proposed to enhance the robustness and accuracy of posture classification, as shown in Figure 6. First, desirable features vectors are extracted from pressure distribution. Both features vectors and pressure distribution are used to classify the postures of the subject. Finally, the outcomes from different classifications are fused to achieve the resultant lying posture.

\subsection{Principal Component Analysis}

Principal Component Analysis (PCA) [38] is a non-parametric method of extracting hidden features from high dimensional data sets (see Figure 7). It transforms complex data set to reveal hidden and simplified underlying structure that cannot be observed directly. The salient spatial features for particular posture can be identified by finding orthogonal linear combinations of principal components (PC)s. The PCs of any data matrix X can be formulated and represented as follows:

$$
\Phi_{i}=\arg \underset{\left\|\Phi_{i}\right\|=1}{\max } \operatorname{var}\left\{\Phi_{i}^{T} X\right\}
$$

In our case, means and variances of the whole pressure distribution over bed are used as basic features for classifying postures. The directions of maximum variance contain the best features for discrimination under Gaussian assumption, and current features possess the most important dynamic of underlying data sets. Although there are many PCs available, selecting optimal number of PCs guarantees minimum loss of information while still retaining important data dynamics. In our analysis, we selected the four most dominant PCs to perform features fusion with Support Vector Machine (SVM) classifiers.

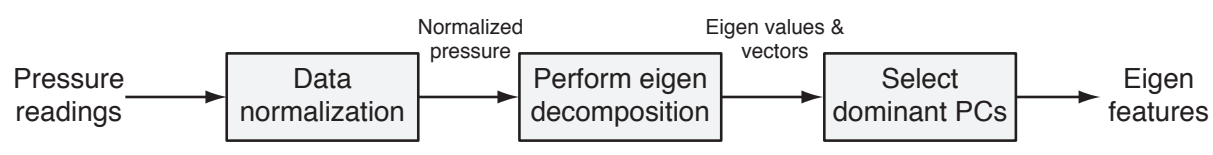

Figure 7. PCA features extraction process. 
By processing and analyzing input pressure readings according to Figure 7, the Eigen features can be extracted to reveal hidden and low dimensional features of specific lying postures through covariance methods. First, normalized pressure matrix, $\mathrm{P}_{\text {bed }}$, with a dimension of $\mathrm{M} \times \mathrm{N}$ are composed.

$$
P_{b e d}=\left[\begin{array}{ccc}
X_{11} & \ldots & X_{1 N} \\
\cdot & \ldots & \cdot \\
X_{M 1} & \ldots & X_{M N}
\end{array}\right]
$$

where $\mathrm{N}$ is a specified number of samples and $\mathrm{M}$ is the desired data width, with their values being 56 and 80 , respectively. The covariance matrix can be calculated as

$$
C_{b e d}=\frac{P_{b e d} P_{b e d}}{M}
$$

Finally, Eigen values and Eigen vectors of resultant covariance matrix can be computed as

$$
C_{b e d} v_{i}=\mu_{i} v_{i}
$$

where $\mu_{\mathrm{i}}$ and $v_{\mathrm{i}}$ are Eigen values and Eigen vectors of covariance matrix, respectively. Finally, the most dominant PC (4 PCs in our case) are selected according to their ranks from ascending orders of Eigen values. The highest Eigen value means the most important dynamics of underlying pressure readings. From the selected PCs, the Eigen features are provided to classifiers to classify the lying posture of the subject.

\subsection{Descriptive Statistics}

Descriptive statistics generally provide basic features of underlying data through simple summaries based on data dispersion and central tendency from pressure distributions. Because of spatial variability of different lying postures on the bed, the applied pressure intensity distributions of different regions are varied statistically. For this reason, the whole bed is divided into nine regions as shown in Figure 8. The number of pressure sensors in a region varies according to the configuration shown in Figure 3. Four descriptive features are calculated in each region to identify spatial features specific to a particular posture. The extracted features include mean $\left(\mathrm{F}_{\text {mean }}\right)$, variance $\left(\mathrm{F}_{\text {variance }}\right)$, standard deviation $\left(\mathrm{F}_{\text {stddev }}\right)$, and Root Mean Square $\left(\mathrm{F}_{\mathrm{rms}}\right)$ values from respective regions. Subsequently, area feature vectors can be determined by combining extracted features from all divided regions. The resultant area feature vectors are modeled with SVM to classify different lying postures. The feature vectors extracted from a set of pressure readings $F_{\text {area }}$ over a specific area of the bed can be expressed as

$$
\mathrm{F}_{\text {area }}(\mathrm{X})=\left[\mathrm{F}_{\text {mean }}(\mathrm{X}), \mathrm{F}_{\text {rms }}(\mathrm{X}), \mathrm{F}_{\text {varience }}(\mathrm{X}), \mathrm{F}_{\text {stddev }}(\mathrm{X})\right]^{\mathrm{T}}
$$

\subsection{Support Vector Machine}

Support Vector Machine (SVM) is basically a non-parametric supervised binary classification technique based on statistical learning theory [40]. It projects original data 


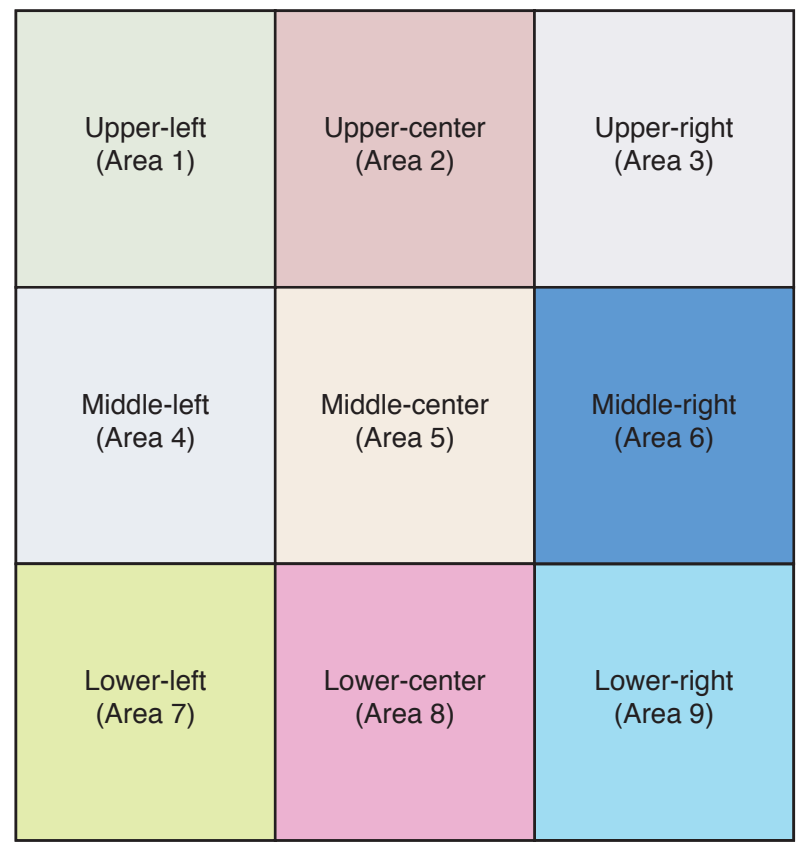

Figure 8. Bed regions for extracting descriptive statistics features.

into a higher dimensional space specified by a kernel function, and computes a maximum-margin hyperplane decision surface that linearly separates the two or more classes. It can achieve best posture classification accuracy from pressure data and features through respective optimal hyperplanes. Our aim is to construct classifier models to predict the lying posture class from unknown data sets in which only attributes are provided. Three different classifiers are trained and constructed according to classifier inputs: pressure intensity data, Eigen features and area statistics features. SVM classification can be presented as the following duality function:

$$
f(x)=\sum \alpha_{i} K\left(x_{i}, x\right)+b
$$

The goal of this duality function is to optimize the margin, $\omega$, constructing a classifier model with canonical separating hyperplanes through the scaled data sets, $\Theta(x i)$ :

$$
\omega=\sum_{i=1}^{m} \alpha_{i} \Theta\left(x_{i}\right)
$$

The procedures of creating posture model with specific kernel parameters and applying it to posture prediction involve offline training and online classification processes as illustrated in Figure 9. The inputs to classifier include pressure data, Eigen features and statistical features. They are independently transformed into scaled data 


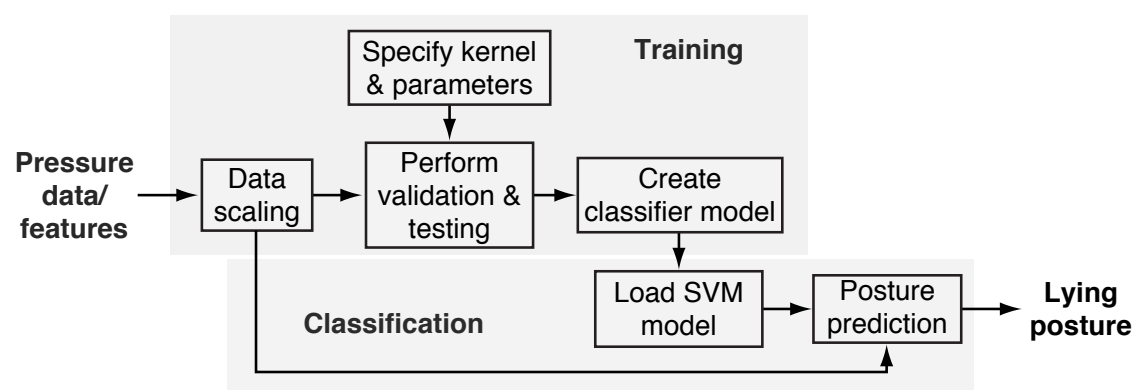

Figure 9. SVM model training and posture classification process.

sets of $[0,+1]$ range with $\mathrm{M}^{\prime}$ variables and $\mathrm{N}^{\prime}$ widths $\left(\mathrm{M}^{\prime} \times \mathrm{N}^{\prime}\right.$ data $)$ as labeled training data sets. The values of M' are 5, 36 and 56 for Eigen statistics, area statistics features and pressure data, respectively. The value of $\mathrm{N}^{\prime}$ is 50 for all three classifiers.

The classifiers are modeled with the appropriate Kernel function and parameters according to the characteristics of underlying data distribution. The following Gaussian kernel, Radial Basis Function (RBF) is used:

$$
K\left(x_{i}, x\right)=\exp \left(-\gamma\left\|x_{i}-x\right\|^{2}\right), \text { where } \gamma=\frac{1}{\sigma^{2}}
$$

Cross-validation and testing is then performed to select the Gaussian kernel parameter, $\gamma$, and regularization cost parameter, $\mathrm{C}$, according to cross-validation accuracy. Different pairs of $\mathrm{C}$ and $\gamma$ were analyzed and the ones with the best posture classification accuracy were selected. It is an iterative process to identify the optimal kernel and classifier parameters with the best posture classification accuracies. The values of $C$ and $\gamma$ pair used in the posture prediction are $\left(2^{3}, 2^{-3}\right)$ and $\left(2^{10}, 2^{-7}\right)$ for both features and data inputs, respectively. Finally, trained classifier models are saved into files to be used in online prediction of posture with unlabeled data sets. To perform online posture classification, trained classifier model is first loaded. Lying posture is predicted based on input pressure features/data. The classifiers' outputs are then fused with weighted voting fusion in order to determine the resultant lying posture.

\subsection{Weighted Voting Fusion}

Due to the high possibility of degradable and erroneous measurements from multiple pressure sensors, uncertainties can be propagated through feature extraction and classification stages. In order to alleviate these problems, intermediate posture results from different classifiers are combined to identify the correct lying posture. Weighted voting is a type of fusion in which some members' votes carry more weight than others through prior empirical measurements [40]. The posture classification accuracy can be enhanced by fusing the intermediate classification outputs from different classifiers to infer the actual lying posture. The posture decision, $\mathrm{D}_{\text {posture, }}$ can be represented as a function of a set of classifiers, $\mathrm{C}_{\mathrm{m}}$ and postures, $\mathrm{P}_{\mathrm{n}}$. The decision can be made by 
selecting the posture with maximum weights by summing weights of the same posture from different classifiers according to the prior assigned posture weight of particular classifier, $W_{C m}^{p}$.

$$
D_{\text {posture }}\left(C_{m}, P_{n}\right)=\arg \max _{p \in n}\left[\sum_{m} W_{C_{m}}^{p}\right]
$$

The weights assigned to the classifiers with respect to lying postures are formulated through classification accuracies obtained from the testing phase. The voting matrix can be built from empirical weights with input classifiers and posture classes. The values of weights in voting matrix are assigned prior to fusion process by analyzing the confusion matrices calculated from different classifiers' outcomes. The assigned weights cannot be altered during the online classification process as their values are dependent on the trained classifier models. Figure 10 illustrates the use of weighted voting to determine the resultant lying posture from SVM classifiers' outputs.

Table 1 shows the voting matrix derived by comparing posture classification and posture misclassification rates with respect to different lying postures. The weights

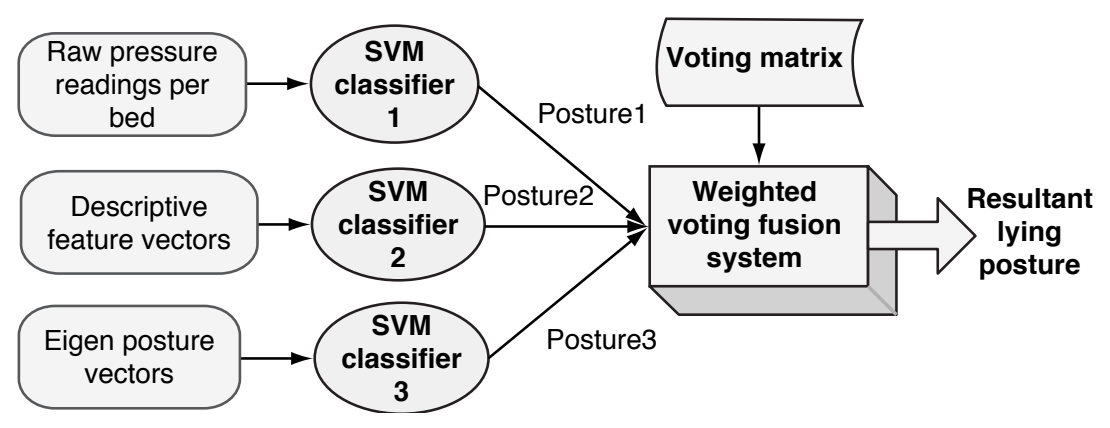

Figure 10. Weighted voting fusion process.

Table 1. Voting fusion matrix with weights assigned to different classifiers and posture classes (data in \%)

\begin{tabular}{llllllllll}
\hline & \multicolumn{8}{c}{ Lying postures } \\
\cline { 2 - 9 } $\begin{array}{c}\text { SVM classifier } \\
\text { types }\end{array}$ & $\mathbf{P}_{\mathbf{1}}$ & $\mathbf{P}_{\mathbf{2}}$ & $\mathbf{P}_{\mathbf{3}}$ & $\mathbf{P}_{\mathbf{4}}$ & $\mathbf{P}_{\mathbf{5}}$ & $\mathbf{P}_{\mathbf{6}}$ & $\mathbf{P}_{\mathbf{7}}$ & $\mathbf{P}_{\mathbf{8}}$ & $\mathbf{P}_{\mathbf{9}}$ \\
\hline $\begin{array}{c}\text { Raw Data } \\
\quad \text { Classifier }\end{array}$ & 25 & 27 & 36 & 33 & 23 & 27 & 20 & 15 & 17 \\
$\begin{array}{c}\text { Statistics Features } \\
\quad \text { Classifier }\end{array}$ & 48 & 39 & 25 & 28 & 39 & 37 & 46 & 45 & 46 \\
$\begin{array}{c}\text { Eigen Features } \\
\quad \text { Classifier }\end{array}$ & 27 & 34 & 39 & 41 & 38 & 36 & 34 & 40 & 37 \\
& & & & & & & & &
\end{tabular}


specified in the matrix are used to identify the resultant lying posture of the subject by taking inputs from different classifiers.

\section{EXPERIMENTAL ANALYSIS AND DISCUSSION}

Since our goal is to develop a monitoring system for lying postures classification through pressure sensitive bed, we conducted posture classification experiments with healthy volunteers using proposed classification approach. The common lying postures considered for experiments include nine postures $\left(\mathrm{P}_{1}\right.$ through $\left.\mathrm{P}_{9}\right)$ as shown in Figure 11 [41]. Six lying postures $\left(\mathrm{P}_{1}{ }_{1}\right.$ through $\left.\mathrm{P}_{6}{ }_{6}\right)$ were identified by grouping some postures of similar spatial characteristics among these nine postures. The postures with minimum spatial differences according to the experiments are regarded as one posture in order to evaluate generalized classification accuracy among different posture classification approaches. For example, left $\log \left(\mathrm{P}_{3}\right)$ and right $\log \left(\mathrm{P}_{4}\right)$ are combined as $\log \left(\mathrm{P}_{3}^{\prime}\right)$; starfish $\left(\mathrm{P}_{7}\right)$, freefaller $\left(\mathrm{P}_{8}\right)$ and soldier $\left(\mathrm{P}_{9}\right)$ are combined as straight $\left(\mathrm{P}^{\prime}{ }_{6}\right)$, as depicted in Figure 11.

\subsection{Experimental Methods}

This study has been approved by the local research ethics committee for protection of human subjects. Five volunteers with height, weight and age of $164.4 \pm 1.4 \% \mathrm{~cm}$ (mean \pm relative standard deviation), $63.2 \pm 15.2 \% \mathrm{~kg}$, and $30 \pm 5.3 \%$ years, respectively, were involved in the experiments conducted at the lab. The pressure measurements of different lying postures with five subjects were collected over three different experiments, resulting in 15 unique data sets. Along with the pressure data, video data were also recorded by a video camera to label pressure data and to validate the posture classification outcomes. In order to avoid having similar data sets and errors propagated from sensors, data collection for each subject was not performed consecutively and the calibration procedure was repeated before each data collection. The influence of sensor's mechanical loading effects such as wear and tear, bending, etc. were minimized through calibration before each experiment. After the experiments, data of three arbitrarily chosen subjects were grouped

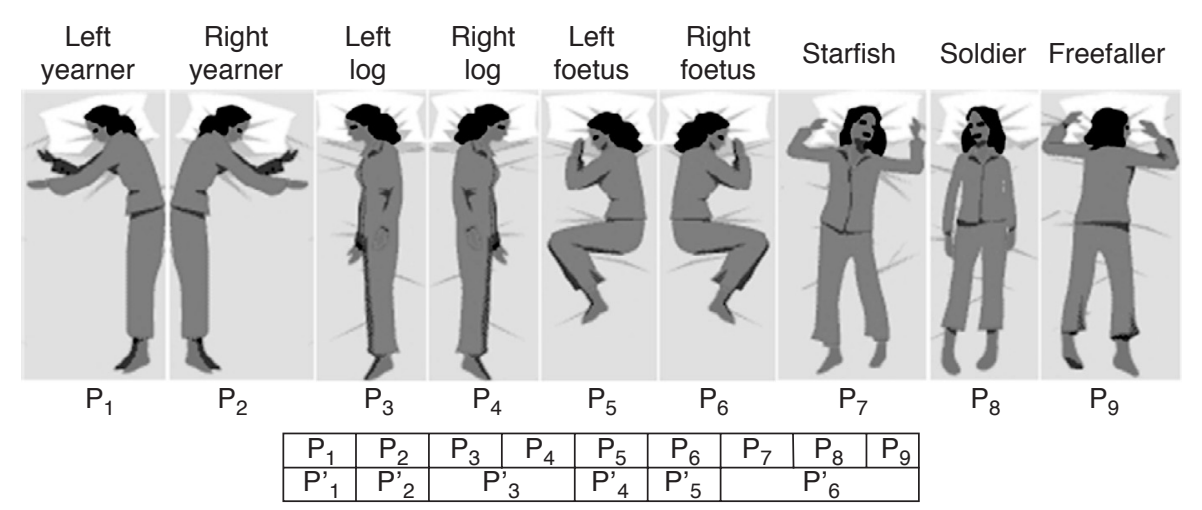

Figure 11. Lying postures considered in posture classification analysis. 
as training data ( 9 data sets), while those of the other two subjects were grouped as testing data (6 data sets). Before applying any feature extraction or classification algorithm, data scaling or normalization was performed on all data sets to make sure that there was no bias in data collected from any subject. Posture classification using SVM classifiers were then applied according to the types of inputs: pressure data, Eigen features and area statistics features. Finally, weighted voting fusion was applied to further enhance the posture classification accuracy by minimizing the misclassification rates.

\subsection{Experimental Results}

Although the analysis was primarily performed with no overlap of data from the same subject in training and testing, we sometimes mixed data of different subjects into testing data. The intention was to test whether the trained classifier model was biased by data from particular training subject. No apparent bias was found in classifying different lying postures. From preliminary classification outcomes, the posture classification accuracies and misclassifications for classifier with normalized pressure data inputs are exhibited in the confusion matrix as shown in Table 2. The same analyses were also conducted for both area statistics features and Eigen features inputs to determine their confusion matrices similar to Table 2.

The confusion matrix shows detailed analysis of classification outcomes from single annotated test data of a particular subject. From similar matrices obtained from different classifiers, the degree of misclassification of postures can be determined. By analyzing the confusion matrices from different classifiers, the weights specified in voting fusion matrix as shown in Table 1 can be determined. Tables 3 and 4 illustrate the classification outcomes from our experiments with different posture classes using different classification approaches. Comparison between Tables 3 and 4 demonstrates that the classification accuracy is improved with fewer posture classes (9 postures vs. 6 postures). The classification accuracies remain the same for all classifiers. This means that the current spatial sensing resolution is still not high enough to classify all possible fine-grained lying postures.

Table 2. Confusion matrix for posture classifier with normalized pressure data input (data in \%)

\begin{tabular}{cccccccccc}
\hline $\mathbf{\%}_{\mathbf{6}}$ & $\mathbf{P}_{\mathbf{1}}$ & $\mathbf{P}_{\mathbf{2}}$ & $\mathbf{P}_{\mathbf{3}}$ & $\mathbf{P}_{\mathbf{4}}$ & $\mathbf{P}_{\mathbf{5}}$ & $\mathbf{P}_{\mathbf{6}}$ & $\mathbf{P}_{\mathbf{7}}$ & $\mathbf{P}_{\mathbf{8}}$ & $\mathbf{P}_{\mathbf{9}}$ \\
\hline $\mathbf{P}_{\mathbf{1}}$ & 87 & 0 & 7 & 2 & 3 & 0 & 0 & 1 & 0 \\
$\mathbf{P}_{\mathbf{2}}$ & 0 & 90 & 0 & 4 & 0 & 3 & 0 & 3 & 0 \\
$\mathbf{P}_{\mathbf{3}}$ & 7 & 0 & 73 & 10 & 0 & 0 & 0 & 8 & 2 \\
$\mathbf{P}_{\mathbf{4}}$ & 0 & 9 & 12 & 65 & 0 & 0 & 0 & 4 & 0 \\
$\mathbf{P}_{\mathbf{5}}$ & 0 & 0 & 0 & 0 & 78 & 0 & 9 & 1 & 12 \\
$\mathbf{P}_{\mathbf{6}}$ & 0 & 5 & 0 & 5 & 0 & 83 & 1 & 2 & 4 \\
$\mathbf{P}_{\mathbf{7}}$ & 0 & 0 & 2 & & 0 & 0 & 88 & 10 & 0 \\
$\mathbf{P}_{\mathbf{8}}$ & 0 & 0 & 2 & 3 & 0 & 0 & 3 & 82 & 10 \\
$\mathbf{P}_{\mathbf{9}}$ & 0 & 0 & 2 & 0 & 5 & 1 & 9 & 23 & 60
\end{tabular}


Table 3. Comparison of classification rates of different approaches using nine lying postures (data in \%)

\begin{tabular}{lccccccccc}
\hline Method & $\mathbf{P}_{\mathbf{1}}$ & $\mathbf{P}_{\mathbf{2}}$ & $\mathbf{P}_{\mathbf{3}}$ & $\mathbf{P}_{\mathbf{4}}$ & $\mathbf{P}_{\mathbf{5}}$ & $\mathbf{P}_{\mathbf{6}}$ & $\mathbf{P}_{\mathbf{7}}$ & $\mathbf{P}_{\mathbf{8}}$ & $\mathbf{P}_{\mathbf{9}}$ \\
\hline $\begin{array}{l}\text { PCA + } \\
\quad 56\end{array}$ & 50 & 53 & 56 & 64 & 60 & 42 & 46 & 40 \\
$\quad$ SVM & 90 & 72 & 70 & 59 & 75 & 86 & 81 & 76 & 63 \\
$\begin{array}{c}\text { Data }+ \\
\quad \text { SVM }\end{array}$ & & & & & & & & & \\
$\begin{array}{l}\text { Statistic }+ \\
\quad \text { SVM }\end{array}$ & 87 & 65 & 71 & 60 & 68 & 72 & 51 & 63 & 45 \\
$\quad$ With Voting & 95 & 92 & 76 & 70 & 89 & 88 & 83 & 80 & 73
\end{tabular}

Table 4. Comparison of classification rates of different approaches using six lying postures (data in \%)

\begin{tabular}{lcccccc}
\hline \multicolumn{1}{c}{ Method } & $\mathbf{P}_{\mathbf{1}}^{\prime}$ & $\mathbf{P}_{\mathbf{2}}$ & $\mathbf{P}_{\mathbf{3}}^{\prime}$ & $\mathbf{P}_{\mathbf{4}}^{\prime}$ & $\mathbf{P}_{\mathbf{5}}{ }^{\prime}$ & $\mathbf{P}_{\mathbf{6}}$ \\
\hline PCA + SVM & 58 & 53 & 64 & 68 & 70 & 75 \\
Data + SVM & 92 & 88 & 81 & 72 & 78 & 90 \\
Statistic + SVM & 95 & 74 & 73 & 78 & 75 & 71 \\
With Voting & 98 & 94 & 93 & 94 & 96 & 92
\end{tabular}

It is noted that the classification between left and right lying postures $\left(\mathrm{P}_{1}\right.$ and $\mathrm{P}_{2}, \mathrm{P}_{3}$ and $\mathrm{P}_{4}, \mathrm{P}_{5}$ and $\mathrm{P}_{6}$ in Table 3; $\mathrm{P}_{1}$ and $\mathrm{P}_{2}, \mathrm{P}_{4}{ }_{4}$ and $\mathrm{P}_{5}{ }_{5}$ in Table 4) did not show similar recognition rates. This may be due to variations of spatiality among subjects. The current results suggest that the classification rate of the posture classifier with normalized data input is generally higher than that of feature-based inputs. As expected, the weighted voting fusion approach using intermediate posture outputs from three classified postures achieves better posture classification outcome than other approaches, as shown in Figure 12.

\subsection{Discussion and Future Directions}

The current experiments show that the mean posture classification accuracy of pressure data input is better than those of features inputs, Eigen features (PCA+SVM) or area statistics features (Statistics + SVM). The extracted features may possibly lose valuable pressure information and hence are unable to correctly differentiate one posture from another. Hence, posture classification with Eigen features can work well with any configuration of sensing map as its classifications are based on spatial compact patterns relevant to particular posture from pressure readings. Although posture classification with area statistics features is able to achieve medium classification rate, its features are completely dependent on spatial dimension and variability of lying posture. The change in orientation of current postures may result in different features and incorrect 


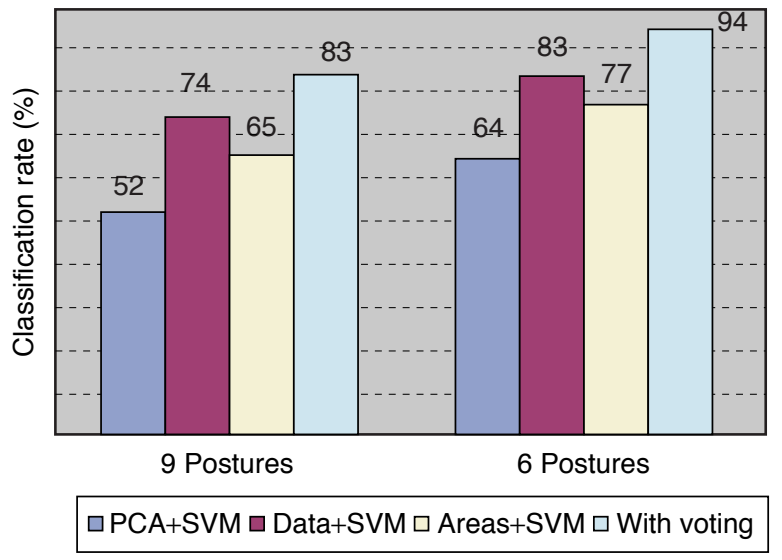

Figure 12. Overall lying posture recognition rates of various approaches over different postures.

classification. Some of the lying postures may not be distinguishable from others due to insufficient spatial difference among different postures although each sensor can provide reliable and accurate measurements. Further analysis is needed to find possible factors affecting the unsymmetrical posture recognition accuracies between left and right symmetric postures. Moreover, the lying postures should not be limited to the current nine and six posture types. It is important to include varieties of different and random lying postures in future experiments to validate our posture classification approach.

Posture classification with direct pressure data worked well with current sensor configuration, but this approach is not easily scalable with high sensor resolution and requires high-performance computing resources. The posture classification using direct pressure data may also not work well when the sensor configuration is considerably changed. The classification accuracies of the PCA+SVM and Statistics+SVM are consistently lower than that of the Data+SVM approach for most of the posture classes. This can be due to the low resolution of the sensor's spatial coverage and the spatial similarity among different lying postures. Table 5 compares the resolution of our pressure sensing bed with others that employ high spatial resolution of sensor coverage

Table 5. Comparison of spatial sensing resolution of pressure sensitive facilities

\begin{tabular}{lccc}
\hline & Total Sensors & Sensing Area, $\mathbf{~ m}^{2}$ & Sensor Resolution, sensors $/ \mathbf{m}^{\mathbf{2}}$ \\
\hline Sensing Chair [33] & 2016 & 0.19 & 10748 \\
Robotic Bed [34] & 221 & 2.00 & 110.5 \\
Our Bed & 56 & 1.62 to 1.8 & 34.6
\end{tabular}


$[36,37]$ and shows the low sensor resolution of our sensing bed. Because of the low spatial sensing resolution of the pressure sensitive bed, the classification accuracies may be dependent on the granularity and type of the postures considered. Hence, we may need to consider validating the current posture classification methods with different numbers and configurations of pressure sensors to examine the relationship between the diversity of postures and resolution. There is no information about comparing the current pressure sensitive bed with high resolution commercial products such as Body Pressure Measurement System (Tekscan Inc, Boston MS, USA) and Pressure Imaging System (XSENSOR Technology Corp, Calgary, Alberta, Canada).

As pressure measurements of real and mockup subjects as well as non-sleeping and actual sleeping conditions have subtle differences, we will validate our system with real patients in actual care settings instead of healthy volunteers measured in the lab. A complete implementation and evaluation is necessary to investigate how lying posture classification and pressure hot spots identification based on interface pressure measurements contribute to assessing potential pressure ulcer risks of the subject. The mean posture classification accuracy of posture outcomes for three classifiers with voting fusion is better than those without voting fusion. An ongoing research effort investigates the performance of multimodal sensors fusion [42] in improving the numbers of successfully identified postures and posture classification accuracies. This may be accomplished by integrating video data into the current implementation for lying posture classification.

\section{CONCLUSION}

This paper presents the design and development of a pressure sensitive bed, iBEPS, featuring lying posture classification with pressure hot spots identification based on interface pressure measurements. Specifically, different methodologies for lying posture classification were evaluated using the pressure sensitive bed. By continuously monitoring the lying posture with interface pressure measurements, potential pressure hot spots of the subject can be identified and appropriate interventions can subsequently be implemented. The design, implementation and evaluation of two-level lying posture classification approach and methodologies are presented in details. Lying posture classification experiments were conducted with the proposed methodologies to evaluate their classification accuracies and limitations, and the results demonstrate that lying postures can be classified up to $93 \%$ accuracy. The current posture classification method may be further enhanced and validated by taking into account random lying postures, testing with different configurations of pressure sensors with real patients in actual care settings, and integrating with inputs from video modality.

\section{NOMENCLATURE}

b The offset of hyperplane from the origin along the unit vector of margin $\omega$

C Cost parameter of SVM that controls the tradeoff between training errors and model complexity

$\mathrm{C}_{\mathrm{bed}} \quad$ Covariance matrix of the normalized pressure matrix

$\mathrm{C}_{\mathrm{m}} \quad$ A set of classifiers considered in weighted voting fusion process 


\begin{tabular}{|c|c|}
\hline $\mathrm{D}_{\text {posture }}$ & Posture decision resulted from weighted voting fusion process \\
\hline$F_{\text {area }}$ & Feature vector of particular area of the bed according to Figure 8 \\
\hline$F_{\text {mean }}$ & Mean pressure measurements calculated from specific area of the bed \\
\hline $\mathrm{F}_{\text {variance }}$ & $\begin{array}{l}\text { Variances of pressure measurements calculated from specific area of } \\
\text { the bed }\end{array}$ \\
\hline $\mathrm{F}_{\text {stddev }}$ & Standard deviation of pressure measurements from specific area of the bed \\
\hline $\mathrm{F}_{\mathrm{rms}}$ & $\begin{array}{l}\text { Root Mean Square (RMS) of pressure measurements from specific area of } \\
\text { the bed }\end{array}$ \\
\hline$K$ & Kernel function of SVM model such as RBF, polynomial, sigmoid, etc. \\
\hline $\mathrm{m}$ & $\begin{array}{l}\text { Total number of classifiers considered in voting fusion process }(\mathrm{m}=3 \text { in our } \\
\text { experiment) }\end{array}$ \\
\hline M & $\begin{array}{l}\text { Total number of rows (number of pressure samples) of input pressure matrix } \\
\text { for PCA }\end{array}$ \\
\hline $\mathbf{M}^{\prime}$ & Dimensions of input data/features of SVM model \\
\hline $\mathrm{n}$ & $\begin{array}{l}\text { Total number of lying posture classes considered in voting fusion process } \\
\text { ( } n=9 \text { for nine postures and } n=6 \text { for six postures in our experiment) }\end{array}$ \\
\hline $\mathrm{N}$ & Total number of columns (data width) of input pressure matrix for PCA \\
\hline $\mathrm{N}^{\prime}$ & Width of data/features vectors of SVM model \\
\hline $\mathrm{P}$ & $\begin{array}{l}\text { Normalized pressure matrix consisting of } \mathrm{M} \times \mathrm{N} \text { pressure measurements } \\
\text { used in PCA }\end{array}$ \\
\hline $\mathrm{P}_{\mathrm{n}}$ & Posture classes considered in weighted voting fusion process \\
\hline$v$ & Eigen vector of the covariance matrix $C_{b e d}$ \\
\hline $\mathrm{W}$ & $\begin{array}{l}\text { Weight assigned to particular posture class and posture classifier used in } \\
\text { voting fusion }\end{array}$ \\
\hline $\mathrm{x}$ & Pressure reading measured from a single pressure sensor \\
\hline $\mathrm{X}$ & A set of pressure readings composed in either vector or matrix form \\
\hline
\end{tabular}

\section{Greek}

Number of support vectors which define the classifier's margin

$\gamma \quad$ Gaussian kernel parameter of SVM classifier with RBF kernel

$\Theta \quad$ Input scaled data set to model SVM classifier

$\mu \quad$ Eigen value of the covariance matrix $\mathrm{C}_{\text {bed }}$

$\omega \quad$ SVM classifier's margin to classify among different posture classes

$\sigma \quad$ Positive number that represents the scaling factor of RBF kernel

$\Phi \quad$ Principal components of input pressure data matrix $\mathrm{X}$

\section{REFERENCES}

[1] A. Mihailidis et al, The $\mathrm{COACH}$ prompting system to assist older adults with dementia through handwashing: An efficacy study, BMC Geriatrics, Vol. 8(28), 2008.

[2] Lebedev MA and Nicolelis MA, Brain-machine interfaces: past, present and future. Trends in Neurosciences, 2006;29(9),536-546.

[3] Zeller et al, Pressure Ulcers, The Journal of the American Medical Association (JAMA), 2006;296(.8), 1020 .

[4] J. Kottner, Pressure Ulcers: A Critical Review of Definitions and Classifications, Ostomy Wound Management, 2009;55(9):22-29. 
[5] European Pressure Ulcer Advisory Panel (EPUAP), Available at: http://www.epuap.org Accessed October 21, 2009.

[6] Berlowitz DR, Brienza DM, Are all pressure ulcers the result of deep tissue injury? A review of the literature. Ostomy/Wound Management. 2007;53,10,34-38.

[7] Park-Lee E et al, Pressure Ulcers Among Nursing Home Residents: United States, 2004, (National Center for Health StatisticsNCHS Data Brief, 2009;14,1-8.

[8] Kaltenhaler E, Whitfield, Walters SJ, Akehurst RL, Paisley S. UK, USA and Canada: how do their pressure ulcer prevalence and incidence data compare? Journal of Wound Care 2001;10(1),530-535.

[9] Bennett G, Dealey C, Posnett J. The cost of pressure ulcers in the UK, Age and Ageing 2004;33(3):230-235.

[10] The Fifth Annual HealthGrades Patients Safety in American Hospitals Study Report, April 2008, Available at: http://www.muschealth.com/ aboutus/PatientSafetyInAmericanHospitalsStudy2008.pdf. Accessed October 21, 2009.

[11] Brian T. Fay et al, What is Interface Pressure?, Proc. of $22^{\text {nd }}$ Conf. of IEEE Engineering in Medicine and Biology Society (EMBS), pp. 2254-2255, July 2000.

[12] Sakai K et al, Continuous Monitoring of Interface Pressure Distribution in Intensive Care Patients for Pressure Ulcer Prevention", Journal of Advanced Nursing, 2009;65(4):809-817.

[13] Gefen, A. Pressure-Sensing Devices for Assessment of Soft Tissue Loading under Bony Prominences: Technological Concepts and Clinical Utilization, Wounds, 2007;19:350-362.

[14] M.D. Stinson, S.A. Crawford and A.P. Porter-Armstrong, Interface pressure measurements: Visual Interpretation of pressure maps with MS clients, Disability and Rehabilitation, 2008;30(8):618-624.

[15] Philips L, Interface Pressure Measurement: Appropriate interpretation of this simple laboratory technique used in thedesign and assessment of pressure ulcer managment device, Australian Wound Management, 2007;15(3):106-113.

[16] K. Bogie et al, new technique for real-time interface pressure analysis: Getting more out of large image data sets, Journal of Rehabilitation Research \& Development, 2008:45(4),523-536.

[17] Reenalda et al, Analysis of healthy sitting behavior: interface pressure distribution and subsutaneous tissue oxygenation, Journal of Rehabilitation Research and Development, 2009,46(5),577-586.

[18] Sprigle S, Dunlop W, Press L, Reliability of bench tests of interface pressure. Assistive Technology, 2003;15:49-576.

[19] J. Reenalda et al, Clinical Use of Interface Pressure to Predict Pressure Ulcer Development: A Systematic Review, Assistive Technology, 2009;21(2):76-85.

[20] A. Gefen. How Much Time Does it Take to Get a Pressure Ulcer? Integrated Evidence from Human, Animal and In Vitro Studies. Ostomy Wound Management, 54:26-35,2008.

[21] A. Gefen A Reswick and Rogers pressure-time curve for pressure ulcer risk. Part 1. Nursing Standard. 2009;23(45),64-74.

[22] A. Gefen A Reswick and Rogers pressure-time curve for pressure ulcer risk. Part 2. Nursing Standard. 2009;23(46),40-44.

[23] Reddy M et al, Preventing Pressure Ulcers: A Systematic Review, The JAMA, 2006;296(8):974-984.

[24] Suriadi et al, Development of a new risk assessment scale for predicting pressure ulcers in an intensive care unit, Nursing in Critical Care, 2008;13(1):34-43.

[25] Mortenson, W.B., \& Miller, W.C.. A review of scales for assessing the risk of developing a pressure ulcer in individuals with SCI. Spinal Cord, 2008;46(3),168-175.

[26] Defloor T and De Schuijmer JD, Preventing Pressure Ulcers: An Evaluation of Four Operating-Table Mattresses, 2000;13(3):134-141.

[27] McInnes E, Cullum NA, Bell-Syer SEM, Dumville JC. Support surfaces for pressure ulcer prevention. Cochrane Database of Systematic Reviews 2008, Issue 3. Art. No.: CD001735. DOI: 10.1002/ 14651858.CD001735.pub3. 
[28] Defloor T, De Bacguer d and Grypdonck MH, The effect of various combinations of turning and pressure reducing devices on the incidence of pressure ulcers, Int. Journal of Nursing Studies, 2005;42(1):37-46.

[29] Inhyuk M. et al, "Control of Air-cell Mattress for Preventing Pressure Ulcer Based on Approximate Anthropometric Model", Proc. of $9^{\text {th }}$ IEEE Conf. on Rehabilitation Robotics, pp.164-167, June-July 2005.

[30] Vanderwee K, Grypdonck M and Defloor T, Alternating pressure air mattresses as prevention for pressure ulcers: A literature review, Int. Journal of Nursing Studies, 2008;45(5):784-801.

[31] A Gefen and J. Levine, The false premise in measuring body-support interface pressures for preventing serious pressure ulcers, Journal of Medical Engineering and Technology, 2007;31(5):375-380.

[32] A. Stekelenburg, D. Gawlitta, D.L. Bader and C.W.J. Oomens, Deep tissue injury: how deep is our understanding?, Arhives of Physical Medicine and Rehabilitation, 2008,89(7),1410-1413.

[33] C. Gehin et al, Which techniques to improve the early detection and prevention of pressure ulcers?, Proc. of the $28^{\text {th }}$ Conf. of EMBS, pp.6057-6060, Aug-Sept 2006.

[34] Wang, Q. et al, Portable Gage for Pressure Ulcer Detection. Proc. of the $28^{\text {th }}$ Conf. of EMBS, pp. 5997-6000, Aug-Sept 2006.

[35] David R. Thomas, Prevention and Treatment of Pressure Ulcers: What works? What doesn't?, The Cleveland Clinic Journal of Medicine 2001,68(8),704-722.

[36] Tan. H.Z. et al, A Sensing Chair using Pressure Distribution Sensors, The IEEE/ASME Transactions on Mechatronics, Vol.6, Issue.3, pp.261-268, Sep 2001.

[37] Kap-Ho Seo et al, Bed-type Robotic System for the Bedridden, Proc. of the IEEE/ASME, pp. 1170-1175, July 2005.

[38] David C. Lay, Linear Algebra and It's Applications, $3^{\text {rd }}$ updated Edition, Addison-Wesley, New York, 2005.

[39] J. S.-Taylor et al, Supprot Vector Machines and other kernel-based learning methods, Cambridge University Press, 2000.

[40] Michal Wozniak, Classifier fusion based on Weighted Voting -Analytical and Experimental Results, Proc. of $8^{\text {th }}$ ISDA, pp.687-692, 2008.

[41] Sleep position gives personality clue, Accessible at: http://news.bbc.co.uk/2/hi/health/3112170.stm. Accessed October 21, 2009.

[42] David L. Hall and James Llinas, Handbook of multisensory data fusion - $3^{\text {rd }}$ Edition, CRC Press, 2001. 



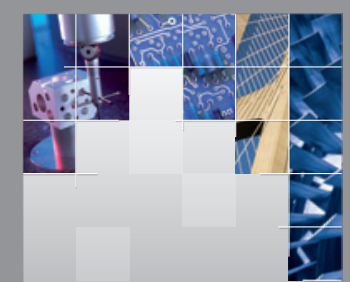

\section{Enfincering}
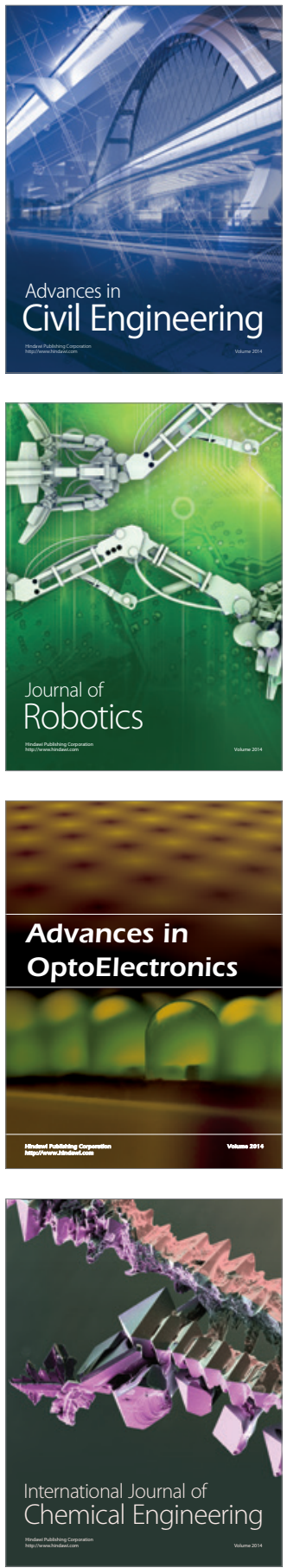

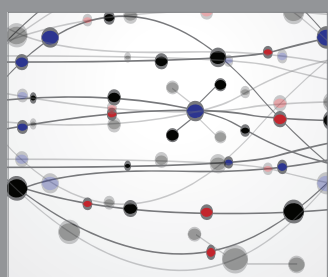

The Scientific World Journal

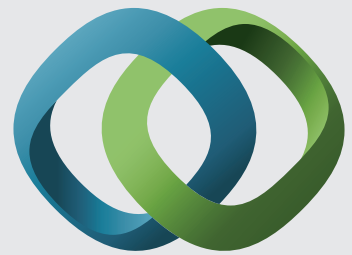

\section{Hindawi}

Submit your manuscripts at

http://www.hindawi.com
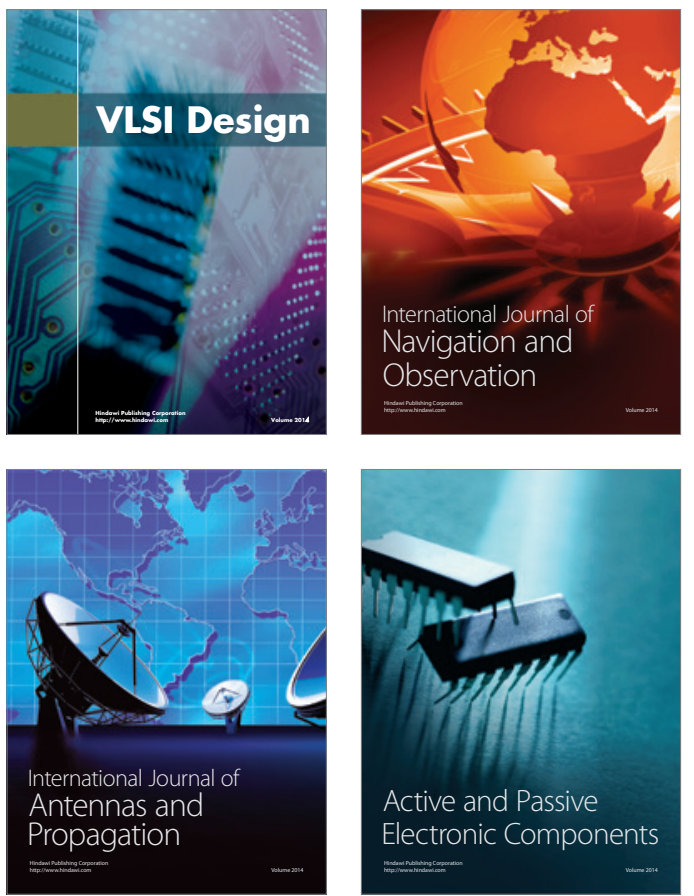
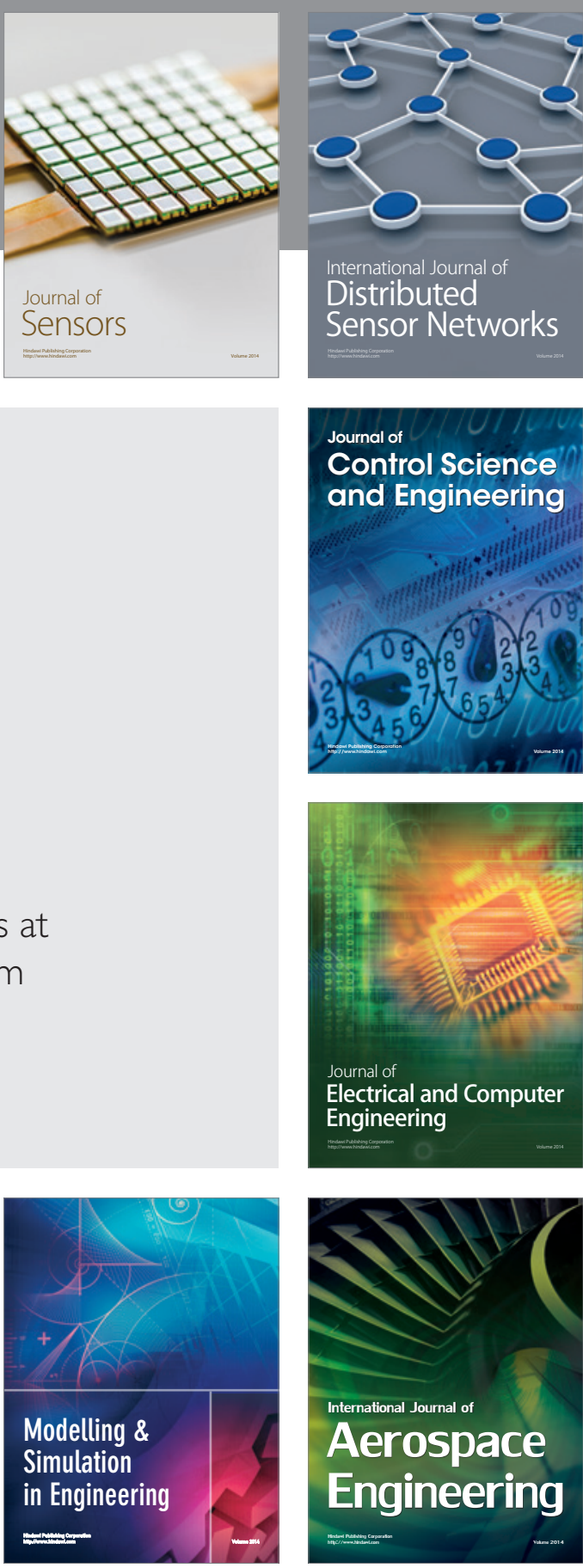

International Journal of

Distributed

Sensor Networks

Journal of

Control Science

and Engineering
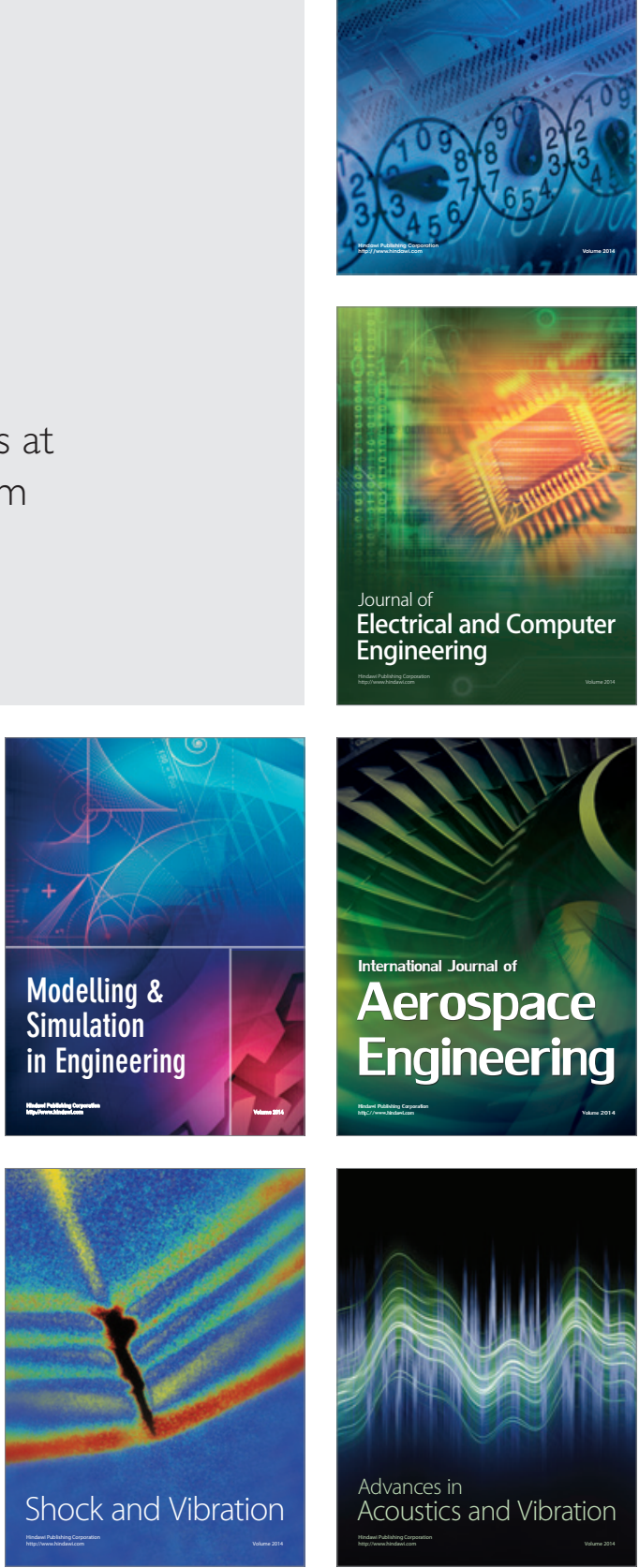